\title{
Dominance and diversity studies of tree species in lesser Himalayan forest of Uttarakhand, India
}

\author{
A.S. BISHT ${ }^{1, \boldsymbol{\varphi}}$, A.B. BHATT ${ }^{2}$ \\ ${ }^{1}$ Department of Basic Science and Humanities, VCSG Uttarakhand University of Horticulture and Forestry, Bharsar 24 6123, Uttarakhand, India, \\ Tel.: +911348-226070, Fax.: +91 1348-226058, ’email: drbishtas@gmail.com \\ ${ }^{2}$ Botany Department, HNB Garhwal University, Srinagar (Garhwal), Uttarakhand 246174, India.
}

Manuscript received: 11 December 2014. Revision accepted: 29January 2016.

\begin{abstract}
Bisht AS, Bhatt AB. 2016. Dominance and diversity studies of tree species in lesser Himalayan forest of Uttarakhand, India. Biodiversitas 17: 70-77. For the present investigation single mountain, approach was applied. This is a supplement the basic approach and extends the gradients further downward in to the forest belt. Vegetational analysis of nine stands covering all the four aspects of the study site of Pauri Garhwal district of Uttarakhand, India has been undertaken. In seven trees, species were encountered. East aspect is characteristics by highest density of Cupressus torulosa while west aspect comprised of Cedrus deodara, Myrica esculenta, Pinus. roxbugrhii, Quercus leucotrichophora and Rhododendron arboreum, i.e.high diversity with low dominance Cupressus torulosa and Cedrus deodara dominated the north aspect. In general, influence of higher anthropogenic pressure on Quercus species is an important factor for leads to gradual replacement of oak species by Pinus roxburghii in all the aspects.
\end{abstract}

Keywords: Diversity, Lesser Himalaya, niche width, regeneration

\section{INTRODUCTION}

Northwest Himalaya is a distinct Himalayan region with a chrematistic climate, geology and flora. The floristic diversity is manifested through different phyto-climatic and topographic regimes. The floral diversity is fascinating because of species richness and diverse community structure. The diversity has occurred in time and space due to a number of ecological changes, often resulting in speciation, isolation, competition, etc. (Gaur et al 2003).

Lesser Himalaya is the central part of Himalaya extending between Siwaliks in south and Great Himalaya in the north and range from $20-60 \mathrm{~km}$ in width. The mountain peak rang between $1200-1300 \mathrm{~m}$ highs where as the valleys range between $400-1200 \mathrm{~m}$. The district Garhwal in northwest Himalaya though represents rich biodiversity, has remained neglected by the past explorers.

The pervasive influence of man in Himalayan forest and heavy dependence of Himalayan agriculture on an "energy subsidy" from the forest (Pandey and Singh 1984) inextricably bind the welfare if Himalayan people to that of the uncultivated ecosystem around them. Accurate measure of the degree of degradation and its effect upon future productivity and quality of lode in the Himalayas is difficult task. Shortage of abiotic resources in the habitat or an excess of a condition unfavorable for function is function known as stress. 'Stress' refer to same environmental situation that often produce changes in organism or ecosystems that we consider being undesirable for example, reduced productivity, an impoverished flora, or an unbalanced species composition stress affects the structure and composition of complex vegetation. Adverse climate, repeated fire, pollution, ionizing radiation
(Woodwell 1970) including local folk (Wiart 1983) are the major mean causing stresses on Himalayan forest.

In any community diversity decrease with increasing stress resulting disappearance of most sensitive species first, then large woody plants and finally all higher plant (mostly woody). Mostly this change occur in natural system as across timberline (Arno and Hammerly 1984), with reduced nutrients (Westman 1975) and where stress has been imposed by man as around metal smelters producing $\mathrm{SO}_{2}$ (Amiro and Courtin 1981). Mostly is observed that the species, which are important for fodder and fuel purpose, are completely eliminated in highly disturbed near settlement sites while unusable species viz. weeds (Eupatorium adenophrum and Parthenium hysterophorum) and shrubs (species of Berberis and Rubus) are frequently distributed near villages.

Damage to individual plants or to forests is accompanied or followed by damage to the productive potential of the land. In areas where stress can be recognized from the vegetation, direct observation of properties of the soil may allow one to estimate the likelihood of permanent damage to its productive capacity. Much nutrient loss may occur in product removal (fodder forest floor litter and dung) during burning or dissolved in run off water and is difficult to detect. However, there can be easily detectable soil losses with out occurrence of the gullies and landslide (Pandey et al 2000).

During the past century, there had been rapid depletion in forest area in whole of the Himalayan region in general and Uttarakhand in particular. The forests of the Lesser Himalayan zone are experiencing the problem of enormous damage to the biological diversity. The factor responsible for the depletion in biodiversity may be attributed to the 
settkenent of villages between 1000 to $2000 \mathrm{~m}$ asl. Most of the needs of villagers are fulfilled from forest, which result in latter's degradation.

\section{MATERIALS AND METHODS}

\section{Study area}

For the present investigation a survey of various summits in district of Pauri Garhwal, Uttarakhand, India, its proximity to site, latitudinal gradient, slope, aspects and other congenial region. The Pauri district lies between Lat. $29^{\circ} 47^{\prime}-30^{\circ} 13^{\prime} \mathrm{N}$ and Long. $78^{\circ} 18^{\prime}-79^{\circ} 10^{\prime} \mathrm{E}$. The study sites exhibited an elevation range from 1800 to 2250 $\mathrm{m}$ (Figure 1). Field research was conducted in two series, i.e. October 2010 to March 2011 and October 2011 to March 2012.

\section{Geomorphology}

District of Pauri Garhwal is one of the thirteen district of Uttarakhand extended in lesser Himalayan zone and known for the hill station. The district is one of the most fascinating segments of the Himalaya, stretches from the Ramganga river that separates Pauti-Kumaun boarder in the east and to the Ganga demarcating the western boarder. Physiographically the study site having undulating topography with gentle slopes in southern and SouthWestern direction (Bisht and Sharma 2014).

\section{Meteorological aspects}

The rainfall pattern in study area is monsoon dependant. The south-east monsoon commences towards the end of June and it rains until mid of September. Northeast monsoon causes occasional winter showering during December to February. The mean monthly rainfall fluctuated between $22.5 \mathrm{~cm}$ to $430 \mathrm{~cm}$ in 2 year. In November of both sampling years, there were no rains. The mean maximum rainfall with maximum number of rainy days in a month was reported in June to September in one or both sampling years (Rawat 2003).

\section{Niche breath}

Niche breath of the $i^{\text {th }}$ species was estimated by the following formula (Levins 1968).

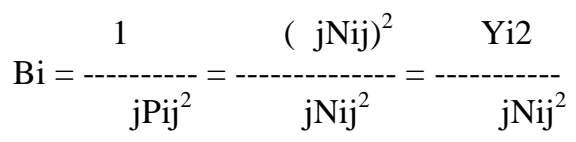

$\mathrm{Bi}=\Sigma \mathrm{j} P \mathrm{Pj} \log \mathrm{Pij}$

Where,

$\mathrm{Nij}=$ Total number of individual of the $\mathrm{i}^{\text {th }}$ species in the resource state.

$\mathrm{Yi}=$ Total number of individuals of the $\mathrm{i}^{\text {th }}$ species over all resource state.

$\mathrm{Pij}=\mathrm{Nij} / \mathrm{Y}=$ Proportion of the individuals of $i^{\text {th }}$ species which is associated with resource state $\mathrm{j}$.

Both the measures are maximized when the species, distributed uniformly over the other resource states. They minimized when the species are associated with only one of the resources state. The measures $\mathrm{Bi}$ and $\mathrm{Bi}$ ' are inverse of Simpson's (1949) measure of concentration and Shannon-Wiener formula (Shannon-Wiener 1949) for formation or uncertainty.

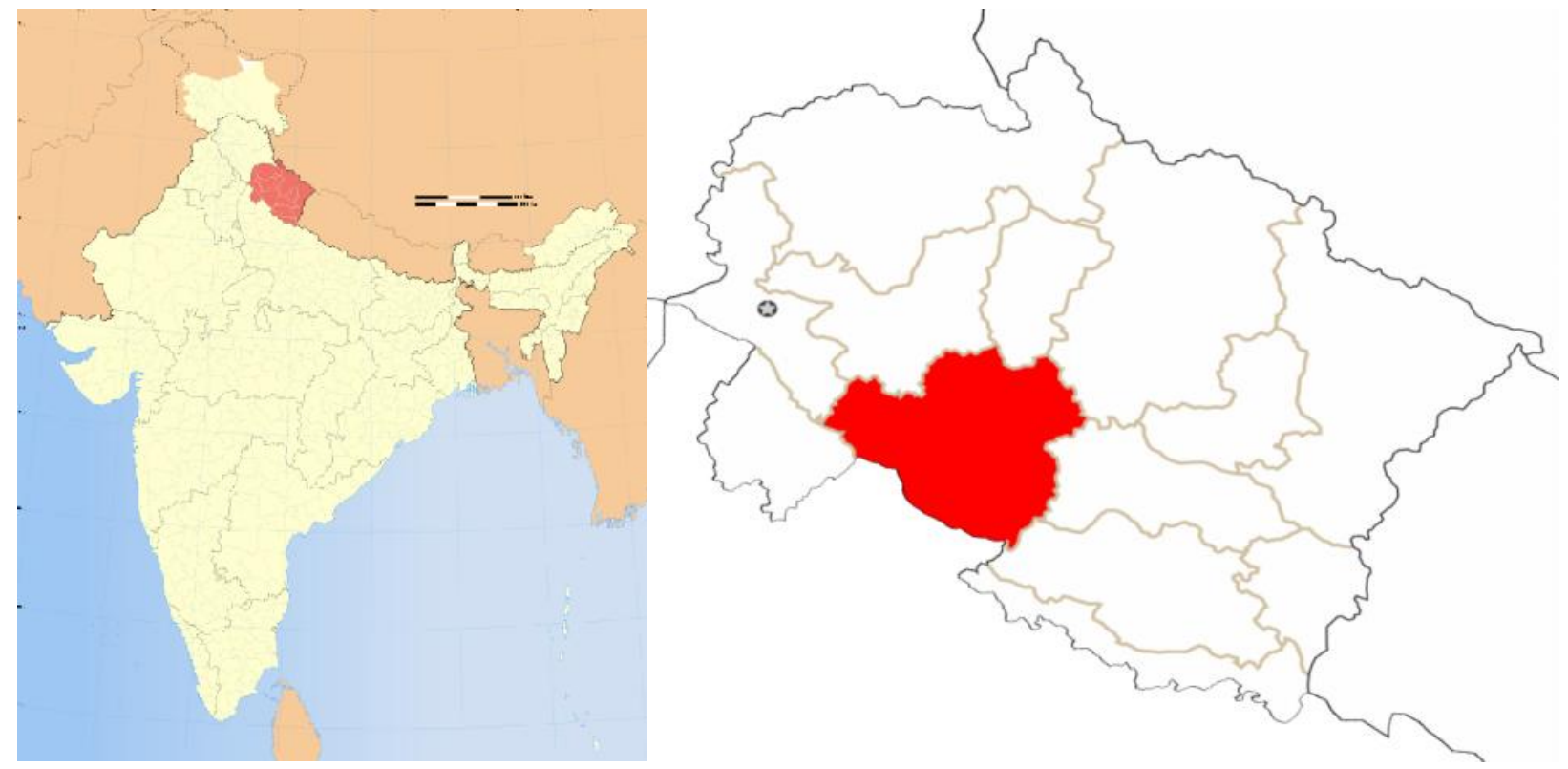

Figure 1. Study area in District of Pauri Garhwal, Uttarakhand, India 


\section{Niche overlap}

The niche overlap between species $\mathrm{i}$ and $\mathrm{h}$ was calculated by the following formula (Colwell and Futuyma 1971).

$$
\mathrm{C}_{\mathrm{ih}}=1-1 / 2 \Sigma_{\mathrm{j}}\left[\mathrm{P}_{\mathrm{ij}}-\mathrm{P}_{\mathrm{hj}}\right]
$$

Where $P_{i j}=N_{i j} / Y_{i}=$ Proportion of the individuals of $i^{\text {th }}$ species which is associated with resource state $\mathrm{j}$ and $\mathrm{P}_{\mathrm{hj}}=$ $\mathrm{N}_{\mathrm{hj}} / \mathrm{Y}$ corresponding to a second species $\mathrm{h}$.

The measure has a minimum value of 0 , when species I and $\mathrm{h}$ share no resource among the resource states. The niche measures (1), (2) and (3) are absolute and are good estimators provider the resources states are all equally distinct. Otherwise, the measures are measures are liable to yield misleading results (Sai and Budholia 1986).

\section{RESULTS AND DISCUSSION}

\section{Quantitative studies: Primary features}

As stated in methodology there were nine stands where the qualitative and quantitative studies were undertaken. Because of quantitative analysis, the vegetation has been studies in terms of mean frequency, density, total basal cover (TBC) and important value index (IVI) of all species. The observations are presented stands wise in Table 1 .

\section{Secondary features}

On the basis of primary data collected directly from the field, the derived attributes have also been worked out for two consecutive year and presented stand wise in Table 2. the features include concentration of dominance generally diversity index, alpha diversity and evenness value of species. Likewise the secondary parameters also include the beta diversity of all the three strata along various stands of the investigation site. The result are presented in Table 3.

\section{Niche width}

Niche width measures the degree of specialization of a species as its ability to exploit an environmental range in space and to maintain its population in different environments. In present investigation the niche breadth ( $\mathrm{Bi}$ and $\mathrm{Bi}$ ') of each species if tree were measured separately at all aspects (east, south, north and west) including submit top (stand $1^{\text {st }}$ ). The niche breath of species was measured aspect wise. Lower and upper elevational stand of each aspect including summit top were considered for computing the niche width of species (Table 4).

Quercus lecotricophora appeared to possess maximum niche breadth at north $(\mathrm{Bi}=2.48$, bi 0.429$)$ and east $(\mathrm{Bi}=$ 1.95. $\mathrm{Bi}$ ' $=0.295$ ) aspect while at south and west aspect it turn out in to $3^{\text {rd }}$ position. $P$. wallichiana had broader niche at east aspect $\left(\mathrm{Bi}=1.80, \mathrm{Bi}^{\prime}=0.276\right)$ while at other aspect it covered minimum niche breath at south aspect $(\mathrm{Bi}=2.67$, Bi'=0.453), the niche breath declined simultaneously at west $\left(\mathrm{Bi}=2.38, \mathrm{Bi}^{\prime}=0.410\right)$ and north aspect $(\mathrm{Bi}=2.0$, $\mathrm{Bi}=0.31)$ followed by west $(\mathrm{Bi}=1.95, \mathrm{Bi}=0.295)$ and north $\left(\mathrm{Bi}=1.54, \mathrm{Bi}^{\prime}=0.233\right)$ and least at east aspect $(\mathrm{Bi}=1.22$,
$\left.\mathrm{Bi}^{\prime}=0.141\right)$. L. ovalifolia had broader niche at south aspect while at other aspects it have minimum value $(\mathrm{Bi}=1.64$, Bi'=0.252) aspect but least at south aspect. $C$. deodara was the only tree species with maximum niche breath at east aspect and it take minimum value at all other aspects.

\section{Niche overlap (Chi)}

The niche overlap value for species of tree on the east south, north and west slopes of the forest of Pauri were calculated separately and presented on Tables 4. Data presented in Table 4 clearly indicate that at east aspect $P$. roxburghii vs $R$. arboreum exhibited maximum niche overlap $(\mathrm{Chi}=0.900)$ followed by $C$. deodara vs $C$. torulosa $(\mathrm{Chi}=0.889)$. Except $C$. torulosa, C.dedara shared minimum resources with other associated species at east slope (Stand $1^{\text {st }}-3^{\text {rd }}$ ). M. esculenta, $P$. roxburghii, $P$. wallichiana, $Q$. lehotrochophora and $R$. arboreum shared huge products of each other as compared to $C$. deodara and C. torulosa.

All plant species occur in a limited range of habitat and with in this range, most of them are more abundant around their particular environmental optimum in the absence of competition (Ter Braak and Prenrice 1988). Species components pf communities thus change along environmental gradients, the replacement and separation of species in the environment depend on variation of resources along these gradients (Pichett 1980).

The Himalayan forest are most productive than the forest of other temperate region with similar rainfall amount, possibly because of a long season of relatively constant favorable temperature and the moderating effect of the mountains during cold winter (Mani 1974). Relatively high wood nutrient concentration produces nutrient accumulation in forest biomass that is relatively higher compared to temperate forest (Singh et al. 1985). It is suggested that forest with multilayer canopy and welldeveloped forest floor would be more productive of soil and water in comparison to a forest having thin layers. Profile diagrams can also be usefully employed in vegetation of lower height to illustrate the relationship between topography and drainage of an area (Kershaw 1973).

The presence of old oak trees and newly developed chir pine patches in the study ate indicates the presence of original oak forest in the area, The study showed the much anthropogenic disturbances in all stands. In a complex Himalayan forest ecosystem chronic from disturbances exists in which people remove only a small fraction of forest biomass in the form of grazing, lopping, surface burning and litter removal at a given time (Khera et al. 2001). These disturbances are affecting the stability of ecosystem and retarding the success ional process in the area. Both natural and human caused disturbances are considered since vegetation responses do not distinguish them between natural and human activities.

The present investigation on vegetational analysis is based upon the comparison of different aspect (N, E, S and $\mathrm{W}$ direction) from the summit top (Stand $1^{\text {st }}$ ). In total 7 species were encountered during whole of the study period at all stands. The finding revels that the high density of $C$. 
Table 1. Mean frequency, density, total basal cover (TBC) and important value index (IVI)

\begin{tabular}{|c|c|c|c|c|c|c|c|c|}
\hline Name of species & $\begin{array}{c}\text { Freq. } \\
\text { 2010-2011 } \\
\end{array}$ & $\begin{array}{c}\text { Freq. } \\
\text { 2011-2012 }\end{array}$ & $\begin{array}{c}\text { Density } \\
\text { 2010-2011 }\end{array}$ & $\begin{array}{c}\text { Density } \\
\text { 2011-2012 }\end{array}$ & $\begin{array}{c}\text { TBC } \\
2010-2011 \\
\end{array}$ & $\begin{array}{c}\text { TBC } \\
2011-2012 \\
\end{array}$ & $\begin{array}{c}\text { IVI } \\
2010-2011 \\
\end{array}$ & $\begin{array}{c}\text { IVI } \\
\text { 2011-2012 } \\
\end{array}$ \\
\hline \multicolumn{9}{|l|}{ Stand I } \\
\hline Cupressus torulosa & 30.00 & 23.33 & 1.4 & 0.8 & 5.68 & 3.23 & 79.35 & 57.24 \\
\hline Lyonia ovalifolia & 10 & $*$ & 0.1 & $*$ & 0.36 & $*$ & 9.56 & $*$ \\
\hline Myrica esculenta & 10 & $*$ & 0.2 & $*$ & 0.14 & $*$ & 8.87 & $*$ \\
\hline Pinus wallichiana & 46.67 & 36.67 & 1.3 & 0.7 & 4.52 & 5.95 & 80.51 & 102.26 \\
\hline Quercus leucotrichophora & 73.33 & 56.67 & 2.1 & 1.5 & 5.13 & 4.34 & 118.45 & 118.17 \\
\hline Rhododendron arboretum & 13.33 & 16.67 & 0.2 & 0.2 & 0.26 & 0.52 & 12.59 & 22.32 \\
\hline \multicolumn{9}{|l|}{ Stand II } \\
\hline Cedrus deodara & 90 & 80 & 3.7 & 2.2 & 19 & 15.23 & 110.1 & 96.95 \\
\hline Cupressus torulosa & 100 & 100 & 8.7 & 7.0 & 36.84 & 41.19 & 187.83 & 203.05 \\
\hline Pinus roxburghii & 10 & $*$ & 0.1 & $*$ & 0.1 & $*$ & 6.22 & $*$ \\
\hline \multicolumn{9}{|l|}{ Stand III } \\
\hline Cedrus deodara & 33 & 13 & 0.8 & 0.2 & 3.96 & 1.06 & 32.52 & 15.90 \\
\hline Cupressus torulosa & 27 & 27 & 0.5 & 0.4 & 1.69 & 1.67 & 21.53 & 28.14 \\
\hline Myrica esculenta & $*$ & 40 & $*$ & 0.7 & $*$ & 1.51 & $*$ & 36.77 \\
\hline Pinus roxburghii & 53 & 40 & 1.0 & 0.7 & 6.22 & 2.95 & 51.07 & 45.74 \\
\hline Pinus wallichiana & 43 & 27 & 0.5 & 0.3 & 4.77 & 1.87 & 34.52 & 26.67 \\
\hline Quercus luecotrichophora & 83 & 70 & 2.6 & 2.7 & 6.99 & 6.94 & 87.43 & 115.8 \\
\hline Rhododendron arboreum & 53 & 40 & 2.3 & 1.2 & 10.45 & 3.03 & 72.93 & 55.49 \\
\hline \multicolumn{9}{|l|}{ Stand IV } \\
\hline Cedrus deodara & 20 & 13.3 & 0.3 & 0.2 & 1.38 & 0.62 & 21.3 & 14.52 \\
\hline Lyonia ovalifolia & $*$ & 20 & $*$ & 0.1 & $*$ & 0.16 & $*$ & 8.94 \\
\hline Myrica esculenta & 30 & 25 & 0.4 & 0.4 & 0.94 & 0.63 & 26.28 & 29.62 \\
\hline Pinus roxburghii & 100 & 86.70 & 5.5 & 5.3 & 32.8 & 28.17 & 235.72 & 242.63 \\
\hline Quercus leucotrichophora & 20 & 15 & 0.3 & 0.3 & 0.3 & 0.38 & 16.7 & 15.38 \\
\hline \multicolumn{9}{|l|}{ Stand V } \\
\hline Lyonia ovalifolia & $*$ & 13.3 & $*$ & 0.1 & $*$ & 0.15 & $*$ & 10.94 \\
\hline Myrica esculenta & 15 & 23.3 & 0.2 & 0.3 & 00.3 & 0.41 & 10.19 & 20.13 \\
\hline Pinus roxburghii & 100 & 100 & 5 & 3.4 & 29.13 & 20.16 & 196.68 & 218.71 \\
\hline Quercus lecotricophora & 66.7 & 33.3 & 1.7 & 0.9 & 3.87 & 2.28 & 65.62 & 47.70 \\
\hline Rhododendron arboreum & 40 & 10 & 0.6 & 0.1 & 1.6 & 0.19 & 30.90 & 7.53 \\
\hline \multicolumn{9}{|l|}{ Stand VI } \\
\hline Cedrus deodara & $*$ & 15 & $*$ & 0.2 & $*$ & 0.41 & $*$ & 14.45 \\
\hline Cupressus torulosa & 20 & 30 & 0.2 & 0.7 & 0.64 & 1.53 & 20.04 & 37.39 \\
\hline Myrica esculenta & 20 & 20 & 0.2 & 0.2 & 0.3 & 0.28 & 16.90 & 17.74 \\
\hline Pinus roxburghii & 93.33 & 83.33 & 3.5 & 3.6 & 18.11 & 76.02 & 209.17 & 220.92 \\
\hline Quercus leucotrichophora & 50 & 26.67 & 0.9 & 0.3 & 1.24 & 2.12 & 53.89 & 26.72 \\
\hline \multicolumn{9}{|l|}{ Stand VII } \\
\hline Myrica esculenta & 10.00 & 53.33 & 0.1 & 0.9 & 0.18 & 1.69 & 6.5 & 84.8 \\
\hline Pinus roxburghii & 33.33 & 50 & 0.9 & 1.1 & 5.38 & 3.9 & 141.38 & 112.61 \\
\hline Quercus leucotrichophora & 56.67 & 43.33 & 1.3 & 1.3 & 3.71 & 2.8 & 156.45 & 102.58 \\
\hline \multicolumn{9}{|l|}{ Stand VIII } \\
\hline Cedrus deodara & 13.33 & 20 & 0.3 & 0.4 & 1.45 & 2.09 & 19.08 & 34.09 \\
\hline Cupressus torulosa & 20 & 20 & 0.4 & 0.2 & 1.99 & 0.99 & 25.3 & 21.56 \\
\hline Myrica esculenta & 50 & 63.33 & 1.0 & 1.0 & 2.67 & 1.61 & 52.66 & 62.46 \\
\hline Pinus roxburghii & 63.33 & 63.33 & 1.5 & 1.0 & 2.62 & 6.21 & 87.29 & 102.44 \\
\hline Quercus leucotrichophora & 33.33 & 30 & 1.0 & 1.3 & 2.46 & 1.16 & 44.72 & 32.23 \\
\hline Rhododendron arboreum & 63.33 & 43.33 & 1.3 & 0.4 & 3.95 & 1.4 & 70.96 & 47.23 \\
\hline \multicolumn{9}{|l|}{ Stand IX } \\
\hline Myrica esculenta & 50 & $*$ & 0.8 & $*$ & 1.63 & $*$ & 69.4 & $*$ \\
\hline Pinus roxburghii & 43.33 & 33.33 & 1.5 & $*$ & 4.21 & 2.18 & 113.53 & 159.53 \\
\hline Quercus leucotrichophora & 33.33 & 10.00 & 0.7 & $*$ & 1.44 & 0.43 & 54.76 & 34.14 \\
\hline Rhododendron arboreum & 36.67 & 23.33 & 1.1 & $*$ & 3.03 & 1.13 & 85.44 & 129.09 \\
\hline
\end{tabular}

Note: $*=$ absent 
Table 2. Concentration of dominance (cd), general diversity index $(\mathrm{H})$, alpha diversity and evenness value of tree species in different season and year at all stands includes gamma diversity

\begin{tabular}{|c|c|c|c|c|c|c|c|c|c|c|}
\hline & \multicolumn{5}{|c|}{ 1st Field research } & \multicolumn{5}{|c|}{ 2nd Field research } \\
\hline & Oct 10 & Jan 11 & Mar 11 & Mean & SD & Oct 11 & Jan 12 & Mar 12 & Mean & SD \\
\hline \multicolumn{11}{|l|}{ Stand I } \\
\hline $\mathrm{Cd}$ & 0.28 & 0.30 & 0.34 & 0.31 & 0.02 & 0.30 & 0.31 & 0.39 & 0.33 & 0.04 \\
\hline $\mathrm{H}$ & 1.40 & 1.32 & 1.18 & 1.30 & 0.09 & 1.27 & 1.24 & 1.09 & 1.20 & 0.08 \\
\hline Alpha & 6.00 & 5.00 & 4.00 & 5.00 & 0.82 & 4.00 & 4.00 & 4.00 & 4.00 & 0.00 \\
\hline Evenness & 1.79 & 1.89 & 1.97 & 1.88 & 0.07 & 2.11 & 2.07 & 1.80 & 1.99 & 0.14 \\
\hline \multicolumn{11}{|l|}{ Stand II } \\
\hline $\mathrm{Cd}$ & 0.50 & 0.52 & 0.56 & 0.53 & 0.02 & 0.58 & 0.55 & 0.57 & 0.57 & 0.01 \\
\hline $\mathrm{H}$ & 0.75 & 0.67 & 0.63 & 0.69 & 0.05 & 0.61 & 0.95 & 0.62 & 0.73 & 0.16 \\
\hline Alpha & 3.00 & 2.00 & 2.00 & 2.33 & 0.47 & 2.00 & 2.00 & 2.00 & 2.00 & 0.00 \\
\hline Evenness & 1.58 & 2.23 & 2.10 & 197 & 0.28 & 2.04 & 3.16 & 2.07 & 2.42 & 0.52 \\
\hline \multicolumn{11}{|l|}{ Stand III } \\
\hline $\mathrm{Cd}$ & 0.19 & 0.22 & 0.23 & 0.21 & 0.02 & 0.17 & 0.25 & 0.29 & 0.24 & 0.05 \\
\hline $\mathrm{H}$ & 1.72 & 1.61 & 1.64 & 1.67 & 0.05 & 1.83 & 2.24 & 1.47 & 1.85 & 0.31 \\
\hline Alpha & 6.00 & 6.00 & 6.00 & 6.00 & 0.00 & 7.00 & 6.00 & 6.00 & 6.33 & 0.47 \\
\hline Evenness & 2.21 & 2.07 & 2.10 & 2.13 & 0.06 & 2.17 & 2.88 & 1.89 & 2.13 & 0.42 \\
\hline \multicolumn{11}{|l|}{ Stand IV } \\
\hline $\mathrm{Cd}$ & 0.63 & 0.62 & 0.65 & 0.63 & 0.01 & 0.68 & 0.64 & 0.69 & 0.67 & 0.02 \\
\hline $\mathrm{H}$ & 0.76 & 0.77 & 0.73 & 0.75 & 0.02 & 0.67 & 0.78 & 0.58 & 0.68 & 0.08 \\
\hline Alpha & 4.00 & 4.00 & 4.00 & 4.00 & 0.00 & 4.00 & 5.00 & 3.00 & 4.00 & 0.82 \\
\hline Evenness & 1.26 & 1.27 & 1.20 & 1.24 & 0.03 & 1.11 & 1.12 & 1.22 & 1.15 & 0.05 \\
\hline \multicolumn{11}{|l|}{ Stand V } \\
\hline $\mathrm{Cd}$ & 0.51 & 0.52 & 0.45 & 0.49 & 0.03 & 0.54 & 0.53 & 0.63 & 0.57 & 0.04 \\
\hline $\mathrm{H}$ & 0.91 & 0.89 & 0.93 & 0.91 & 0.02 & 0.82 & 0.96 & 0.74 & 0.84 & 0.09 \\
\hline Alpha & 4.00 & 4.00 & 3.00 & 3.67 & 0.47 & 4.00 & 5.00 & 4.00 & 4.33 & 0.47 \\
\hline Evenness & 1.52 & 1.48 & 1.94 & 1.64 & 0.21 & 1.37 & 1.37 & 1.23 & 1.32 & 0.06 \\
\hline \multicolumn{11}{|l|}{ Stand VI } \\
\hline $\mathrm{Cd}$ & 0.43 & 0.60 & 0.58 & 0.54 & 0.08 & 0.51 & 0.42 & 0.79 & 0.57 & 0.16 \\
\hline $\mathrm{H}$ & 1.04 & 0.79 & 0.82 & 0.89 & 0.11 & 1.00 & 1.15 & 0.43 & 0.86 & 0.31 \\
\hline Alpha & 4.00 & 4.00 & 4.00 & 4.00 & 0.00 & 5.00 & 5.00 & 3.00 & 4.33 & 0.94 \\
\hline Evenness & 1.73 & 1.32 & 1.37 & 1.47 & 0.19 & 1.44 & 1.65 & 0.90 & 1.33 & 0.31 \\
\hline \multicolumn{11}{|l|}{ Stand VII } \\
\hline $\mathrm{Cd}$ & 0.54 & 0.55 & 0.51 & 0.53 & 0.02 & 0.34 & 0.34 & 0.35 & 0.34 & 0.00 \\
\hline $\mathrm{H}$ & 0.72 & 0.64 & 0.68 & 0.68 & 0.04 & 1.09 & 1.09 & 1.08 & 1.09 & 0.01 \\
\hline Alpha & 3.00 & 2.00 & 2.00 & 2.33 & 0.47 & 3.00 & 3.00 & 3.00 & 3.00 & 0.00 \\
\hline Evenness & 1.52 & 2.12 & 2.26 & 1.97 & 0.32 & 2.29 & 2.29 & 2.26 & 2.28 & 0.01 \\
\hline \multicolumn{11}{|l|}{ Stand VIII } \\
\hline $\mathrm{Cd}$ & 0.20 & 0.20 & 0.21 & 0.20 & 0.00 & 0.20 & 0.24 & 0.22 & 0.22 & 0.02 \\
\hline $\mathrm{H}$ & 1.68 & 1.68 & 1.66 & 1.67 & 0.01 & 1.70 & 1.58 & 1.66 & 1.65 & 0.05 \\
\hline Alpha & 6.00 & 6.00 & 6.00 & 6.00 & 0.00 & 6.00 & 6.00 & 6.00 & 6.00 & 0.00 \\
\hline Evenness & 2.16 & 2.16 & 2.13 & 2.15 & 0.02 & 2.18 & 2.03 & 2.14 & 2.11 & 0.07 \\
\hline \multicolumn{11}{|l|}{ Stand IX } \\
\hline $\mathrm{Cd}$ & 0.39 & 0.26 & 0.26 & 0.30 & 0.06 & 0.52 & 0.51 & 0.50 & 0.51 & 0.01 \\
\hline $\mathrm{H}$ & 1.01 & 1.37 & 1.37 & 1.25 & 0.17 & 0.83 & 0.69 & 0.69 & 0.74 & 0.07 \\
\hline Alpha & 3.00 & 4.00 & 3.00 & 3.33 & 0.47 & 3.00 & 2.00 & 2.00 & 2.33 & 0.47 \\
\hline Evenness & 2.11 & 2.28 & 2.86 & 2.42 & 0.32 & 1.75 & 2.29 & 2.30 & 2.11 & 0.26 \\
\hline
\end{tabular}

torulasa forest $\left(87.0\right.$ trees $\left.100 \mathrm{~m}^{-2}\right)$ was observed on the east (Cooler)aspect and the lower zone (stand $2^{\text {nd }}$ ) where it was found associated with $C$. deodara only. The lowest density of $C$. torulosa $\left(2.0\right.$ trees $\left.\mathrm{m}^{-2}\right)$ was observed on the west aspect ( $\operatorname{stand} 8^{\text {th }}$ ) where it was associated with $C$. deodara, $M$. esculenta, $P$. roxurghii, $Q$. leucotrichophora and $R$. arboreum which is supported by the fact that high diversity of plants decreases the dominance if species. In the south aspect (stands $4^{\text {th }}$ and $5^{\text {th }}$ ) this species was completely absent. C. torulosa emerged as a co-dominant 
Table 4. Niche overlap between species of trees at all aspects

\begin{tabular}{|c|c|c|c|c|c|c|c|c|c|}
\hline S.N. & Name of species & $\begin{array}{l}1 \\
\text { Cd }\end{array}$ & $\begin{array}{l}2 \\
\mathrm{Ct}\end{array}$ & $\begin{array}{l}3 \\
\text { Lo }\end{array}$ & $\begin{array}{l}4 \\
\text { Me }\end{array}$ & $\begin{array}{l}5 \\
\text { Pr } \\
\end{array}$ & $\begin{array}{l}6 \\
\text { Pw }\end{array}$ & $\begin{array}{l}7 \\
\text { QI }\end{array}$ & $\begin{array}{l}\mathbf{8} \\
\mathbf{R a}\end{array}$ \\
\hline \multicolumn{10}{|c|}{ East aspect } \\
\hline 1 & Cedrus deodara & $*$ & & & & & & & \\
\hline 2 & Cupressus torulosa & 0.889 & $*$ & & & & & & \\
\hline 3 & Lyonia ovalifolia & 0.000 & 0.112 & $*$ & & & & & \\
\hline 4 & Myrica esculenta & 0.152 & 0.152 & 0.22 & $*$ & & & & \\
\hline 5 & Pinus roxburghii & 0.252 & 0.141 & 0.00 & 0.778 & $*$ & & & \\
\hline 6 & Pinus wallichiana & 0.152 & 0.152 & 0.667 & 0.556 & 0.333 & $*$ & & \\
\hline 7 & Qurecus leucotrichophora & 0.152 & 0.152 & 0.420 & 0.802 & 0.58 & 0.753 & $*$ & \\
\hline 8 & Rhododendron arboreium & 0.152 & 0.141 & 1.00 & 0.878 & 0.900 & 0.433 & 0.68 & $*$ \\
\hline \multicolumn{10}{|c|}{ South aspect } \\
\hline 1 & Cedrus deodara & $*$ & & & & & & & \\
\hline 2 & Cupressus torulosa & 0.000 & $*$ & & & & & & \\
\hline 3 & Lyonia ovalifolia & 0.600 & 0.200 & $*$ & & & & & \\
\hline 4 & Myrica esculenta & 0.500 & 0.250 & 0.900 & $*$ & & & & \\
\hline 5 & Pinus roxburghii & 0.497 & 0.000 & 0.679 & 0.729 & $*$ & & & \\
\hline 6 & Pinus wallichiana & 0.000 & 1.000 & 2.000 & 0.250 & 0.000 & $*$ & & \\
\hline 7 & Qurecus leucotrichophora & 0.091 & 0.636 & 0.491 & 0.591 & 0.364 & 0.606 & $*$ & \\
\hline 8 & Rhododendron arboreum & 0.000 & 0.333 & 0.400 & 0.500 & 0.521 & 0.333 & 0.606 & $*$ \\
\hline \multicolumn{10}{|c|}{ North aspect } \\
\hline 1 & Cedrus deodara & $*$ & & & & & & & \\
\hline 2 & Cupressus torulosa & 0.267 & $*$ & & & & & & \\
\hline 3 & Lyonia ovalifolia & 0.000 & 0.365 & $*$ & & & & & \\
\hline 4 & Myrica esculenta & 0.182 & 0.003 & 0.182 & $*$ & & & & \\
\hline 5 & Pinus roxburghii & 0.773 & 0.407 & 0.000 & 0.409 & $*$ & & & \\
\hline 6 & Pinus wallichiana & 0.000 & 0.367 & 1.000 & 0.182 & 0.000 & $*$ & & \\
\hline 7 & Qurecus leucotrichophora & 0.150 & 0.675 & 0.525 & 0.657 & 0.377 & 0.525 & $*$ & \\
\hline 8 & Rhododendron arboreum & 0.000 & 0.733 & 1.000 & 0.182 & 0.000 & 1.000 & 0.525 & $*$ \\
\hline \multicolumn{10}{|c|}{ West aspect } \\
\hline 1 & Cedrus deodara & $*$ & & & & & & & \\
\hline 2 & Cupressus torulosa & 0.214 & $*$ & & & & & & \\
\hline 3 & Lyonia ovalifolia & 0.000 & 0.786 & $*$ & & & & & \\
\hline 4 & Myrica esculenta & 0.500 & 0.314 & 0.100 & $*$ & & & & \\
\hline 5 & Pinus roxburghii & 0.583 & 0.214 & 0.000 & 0.900 & $*$ & & & \\
\hline 6 & Pinus wallichiana & 0.000 & 0.786 & 1.000 & 0.100 & 0.00 & $*$ & & \\
\hline 7 & Qurecus leucotrichophora & 0.206 & 0.824 & 0.618 & 0.618 & 0.383 & 0.618 & $*$ & \\
\hline 8 & Rhododendron arboreium & 0.524 & 0.310 & 0.095 & 0.095 & 0.905 & 0.095 & 0.478 & $*$ \\
\hline
\end{tabular}

Table 3. Beta diversity matrices for species

\begin{tabular}{llllllllll}
\hline Stands & $\mathbf{1}$ & $\mathbf{2}$ & $\mathbf{3}$ & $\mathbf{4}$ & $\mathbf{5}$ & $\mathbf{6}$ & $\mathbf{7}$ & $\mathbf{8}$ & $\mathbf{9}$ \\
\hline 1 & $*$ & & & & & & & & \\
2 & 0.00 & $*$ & & & & & & & \\
3 & 1.24 & 0.95 & $*$ & & & & & & \\
4 & 0.73 & 0.53 & 1.03 & $*$ & & & & & \\
5 & 1.10 & 0.00 & 1.03 & 1.20 & $*$ & & & & \\
6 & 0.73 & 1.07 & 1.37 & 1.20 & 0.80 & $*$ & & & \\
7 & 1.10 & 0.00 & 0.95 & 1.07 & 1.07 & 1.07 & $*$ & & \\
8 & 1.00 & 1.00 & 1.55 & 1.10 & 1.10 & 1.47 & 1.00 & $*$ & \\
9 & 0.83 & 0.00 & 1.18 & 0.90 & 1.35 & 0.90 & 1.17 & 1.25 & $*$ \\
\hline
\end{tabular}

species at stand $2^{\text {nd }}$ (east aspect) it had maximum density (37.0 trees $\left.100 \mathrm{~m}^{-2}\right)$, TBC $\left(19.0 \mathrm{~m}^{2}\right)$ and frequency $(90 \%)$ in this stand $\left(2^{\text {nd }}\right)$ followed by sites having west and north aspect. In the north aspect the presence of $C$. torulosa and
C. deodara was due to the plantation program undertaken by the forest department. The forest of $C$. deodara can be literally attributed to edaphic and topographical conditions. The development of deodar forest is associated with residual soil formation (Joshi et al. 1983) being the east face, the low insolations and high moisture condition of slope further promoter its growth. $Q$. leucotricophora was found in all stands except stand $2^{\text {nd }}$. It is dominated in the summit top (Stand $1^{\text {st }}$ ) having maximum IVI value (118.45). Further it is more frequent in the upper elevation of east and north face, while in the west aspect its occupied small TBC $\left(0.30-0.38 \mathrm{~m}^{2} 100 \mathrm{~m}^{-2}\right)$ and exhibits least density (3.0 trees $100 \mathrm{~m}^{-2}$ ) as compared to P. roxburghii. Due the fact that the lower stands of north and south aspect were dominated by chir pine while the east and west aspects comprise $C$. deodara, $C$. torulosa, $R$. arboreum, etc. in the upper elevation of these aspects were dominated by $P$. roxburghii. Influence the higher 
Table 4. Niche breath in all aspects

\begin{tabular}{llll}
\hline S.N. & Name of species & Bi & Bi \\
\hline East aspect & & \\
1 & Cedrus deodara & 1.35 & 0.185 \\
2 & Cupressus tolurosa & 1.36 & 0.223 \\
3 & Lyonia ovalifolia & 1.00 & 0.00 \\
4 & Myrica esculanta & 1.53 & 0.23 \\
5 & Pinus roxburghii & 1.22 & 0.141 \\
6 & Pinus wallichiana & 1.80 & 0.276
\end{tabular}

\section{South aspect}

$\begin{array}{llll}1 & \text { Cedrus deodara } & 1.00 & 0.00 \\ 2 & \text { Cupressus torulosa } & 1.00 & 0.00 \\ 3 & \text { Myrica esculenta } & 2.67 & 0.452 \\ 4 & \text { Pinus roxburghii } & 2.00 & 0.301 \\ 5 & \text { Pinus wallichiana } & 1.00 & 0.00 \\ 6 & \text { Quercus luechotrichophora } & 2.05 & 0.373 \\ 7 & \text { Rhododendron arboreum } & 1.80 & 0.276\end{array}$

\section{North aspect}

$\begin{array}{llll}1 & \text { Cedrus deodara } & 1.00 & 0.00 \\ 2 & \text { Cupressus torulosa } & 1.64 & 0.252 \\ 3 & \text { Lyonia ovalifolia } & 1.00 & 0.00 \\ 4 & \text { Myrica esculenta } & 2.12 & 0.394 \\ 5 & \text { Pinus roxburghii } & 1.54 & 0.233 \\ 6 & \text { Pinus wallichiana } & 1.00 & 0.00 \\ 7 & \text { Quercus leucotrichophora } & 2.48 & 0.429 \\ 8 & \text { Rhododendron arboreum } & 1.00 & 0.00\end{array}$

\section{West aspect}

\begin{tabular}{llll}
1 & Cedrus deodara & 1.00 & 0.00 \\
2 & Cupressus torulosa & 1.51 & 0.226 \\
3 & Lyonia ovalifolia & 1.00 & 0.000 \\
4 & Myrica esculenta & 2.38 & 0.410 \\
5 & Pinus roxburghii & 1.95 & 0.295 \\
6 & Pinus wallichiana & 1.00 & 0.000 \\
7 & Quercus leucotrichophora & 2.20 & 0.404 \\
8 & Rhododendron arboreium & 2.33 & 0.404 \\
& & & \\
Landscape level & & \\
1 & Cedrus deodara & 2.020 & 0.451 \\
2 & Cupressus torulosa & 1.560 & 0.333 \\
3 & Lyonia ovalifolia & 2.270 & 0.414 \\
4 & Myrica esculenta & 6.080 & 0.831 \\
5 & Pinus roxburghii & 5.340 & 0.785 \\
6 & Pinus wallichiana & 1.800 & 0.276 \\
7 & Quercus leucotrichophora & 5.320 & 0.804 \\
8 & Rhododendron arboreum & 3.500 & 0.604 \\
& & & \\
\hline
\end{tabular}

anthropogenic pressure on this species us another important cause for presence of lower number of both small and large oak tree in the study area. Large number of tree are chopped and lopped for fodder, fuel purpose and log for construction work, resulting more open canopy which provide for favorable environmental condition for the invasion of secondary species. Chettri et al. (2002) observed that in both open canopy and closed canopy forest, reduced value for IVI and basal area for species that are preferred for fire wood. The chir pine ( $P$. roxburghii) enjoyed as a dominant species at all aspect completely absent at the summit (stand $1^{\text {st }}$ ) with highest elevation
(2300 $\mathrm{m}$ asl). As compared to other stands, it was less common in the east aspect as Pinus grows more rapidly in the drier area as compared to cool moist areas. This situation is comparable with the studies done by Singh and Singh (1992) and Singh et al.(1997) on Kumaun Himalaya and Sharma and Baduni (2000) in the moist temperate forest of Garhwal Himalaya and Sundriyal and Sharma (1996), Chetrii et al(2002) on Sikkim Himalaya. Summit top (stand $1^{\text {st }}$ ) possess the maximum tree species richness and represents the trees of all other aspect (except $P$. roxburghii), supported the fat that higher altitudes promotes heterogeneity. Summit point itself gives the idea of general vegetation pattern. In its east aspect was $C$. deodara, $C$. torulosa and $R$. arboreum. The south facing slope of this stands was burned by the forest fire but the dry tree of C.torulosa, C. deodara and Q. leucotrichophora give an idea about the closed forest patch in the past.

Bhandari et al. (1998), Ghidiyal et al. (1998), Bankoti and Tewari (2001), Khera et al. (2001) etc. workers reported the similar pattern of species diversity in distributed forest of Central Himalaya with special reference to aspect and altitude. The present finding for diversity index falls well with in the range of other temperate forests. Monk (1967) and Risser and Rice (1971) obtained 2.3 as the highest value for diversity index for temperate vegetation including forest trees. Barun (1950) reported species diversity between 1.69 and 3.40 in an eastern deciduous forest vegetation including herbs at North America. Baduni and Sharma (1997) reported diversity index value up to 1.70 for moist temperate forest of Garhwal Himalaya. On the other hand, tropical forest including vegetation of Savana indicate higher diversity index as calculated by Knight (1975) for young $(\mathrm{H}=5.06)$ and old $(\mathrm{H}=5.40)$ stands.

A second major component of diversity is evenness or equitability in the apportionment of individuals among the species. The evenness varied between 1.26 (south aspect) to 3.16 (east aspect). Moist cooler conditions, moderate soil temperature and lower degree of human disturbance are the main factor for the equal share of individuals among species at east aspect. The individual of species in south and north aspects were not equally distributed due to the microclimatic difference and varying anthropogenic disturbance.

The analysis of niche relationship in natural communities is of considerable interest. The way in which species with in ecological communities partitions available resources among themselves is a major determinant of the diversity of co-existing species (MacArthur 1958). All else being equal, a community, with more resources sharing, or greater niche overlap, will clearly support more species than one with less niche overlap (Pianka 1974).

\section{REFERENCES}

Amiro BD, Courtin GM. 1981. Pattern of vegetation in the vicinity of an industrially disturbed ecosystem, Sudbury, Ontario. Canadian J Bot 59: $1623-1639$.

Arno SF, Hammerly RP. 1984. Timberline. Mountain and Arctic forest frontiers. Mountaineers, Seattle, Washington. 
Baduni NP, Sharma CM. 1997. Flexibility-fitness-compromise of the trees in moist temperate forest of Garhwal Himalaya. Ann For 5 (2): 126135.

Bankoti NS, Tewari LM. 2001. Analysis of forest vegetation at and around Soni-Binsar area in Kumaun Himalaya. In: Pande PC, Samant SS (ed.). Plant Diversity of the Himalaya. Gyanodaya Prakashan, Nainital.

Bhandari BS, Metha JP, Tiwari SC. 1998. Woody vegetation structure along an altitudinal gradient in a part of Garhwal Himalaya. J Hill Res 11 (1): 26-31.

Bisht AS, Sharma KD. 2014. Plants utilization by the communities of Bharsar and adjoining area of Pauri Garhwal District, Uttarakhand, India. Biodiversitas 15 (1): 94-100

Braun EL. 2000. The ecology of the forest of Eastern North America, their development, composition and distribution. Deciduous forest of Eastern North America. McGraw Hill, New York.

Chettri NE, Klabya DC, Sundriayal RC. 2002. Impact of firewood extraction on tree structure, regeneration and woody biomass productivity in a trekking corridor of the Sikkim Himalaya. Mountain Res Dev 22 (2): 150-158.

Colwell RK, Futuyma DJ. 1971. On the measurement of niche breadth and overlap. Ecology 52: 567-576.

Connell JH, Orias E. 1964. The ecological regulation of species diversity. Amer Nat 98: 399-414.

Gaur UN, Raturi GP, Bhatt AB. 2003. Quantitative response of vegetation in glacial moraine of Central Himalaya. The Environmentalist (23): 237-247.

Ghidiyal S, Baduni NP, Khanduri VP, Sharma CM. 1998. Community structure and composition of oak forests, along altitudinal gradients in Garhwal Himalaya, Indian J For 21 (3): 242-247.

Joshi SC, Joshi DR, Dani DD. 1983. Kumaun Himalaya- A Geographica Perspective on Resource Development. Gyanodaya Prakashan, Nainital

Kershaw KK. 1973. Quantitative and Dynamic Plant Ecology. 2nd ed ELBS and Edward Arnold (Publ.) Ltd. London.

Khera N, Kumar A, Ram J, Tewari A. 2001. Plant biodiversity assessment in relation to disturbances in mid-elevational forests of Central Himalaya, India. Trop Ecol 42 (1): 83-95.

Knight DH. 1975. A phytosociological analysis of species rich tropical forest on Barro Colorado Island, Panama. Ecol Monogr 45: 259-284.

Levins R. 1968. Evolution of changing environments: Some theoretical explorations. Amer Nat 147: 784-812.

MacArthur RH. 1958. Population ecology of some warblers of Northeastern coniferous forests. Ecology 39 (4): 599-619.

Mani MS. 1974. Ecology and Biogeography of High Altitudes. Insects Junk Publishers. The Hague.
Monk CD. 1967. Tree species diversity in the eastern deciduous forest with particular reference to North Central Florida. Am Nat 101: 173187.

Pandey AN. Singh JS. 1984. Mechanism of ecosystem recovery: A case study from Kumaun Himalaya. Reclam Reveg Res 3: 271-292

Pandey N, Nautiyal BP, Bhatt AB. 2000. Studies on vegetation analysis, plant form and biological spectrum of an alpine zone of north-west Himalaya. Trop Ecol, 40: 163-166

Pianka ER. 1974. Niche overlap and diffuse competition. Proc Natl Acad Sci USA 71: 2141-2145.

Pichett STA. 1980. Non equilibrium co existence of plants. Bull Theor Bot Club 107: 238-248.

Rawat J. 2003. Completion, Niche and Diversity patterns along an altitudinal gradient in a part of lesser Himalaya in Uttaranchal. [Dissertation]. HNB Garhwal University, Srinagar, India

Risser PG, Rice EL. 1971. Diversity in tree species in Oklahoma upland forest. Ecology 52: 876-880.

Sai VS, Budholia SS. 1986. Niche measurement of tree species in central India forest. Trop Ecol 27: 76-84.

Shannon CE, Wiener W. 1949. The mathematical theory of communication. University of Illinois Press, Urbana, IL.

Sharma CM, Baduni NP. 2000. Effect of aspect on the structure of some natural stands of Abies pindrow in Himalayan moist temperate forest. The Environmentalist 20: 309-317.

Simpson EH. 1949. Measurement of diversity. Nature 163: 688.

Singh JS, Singh SP 1992. Forests of Himalaya: Structure, functioning and impact of Man. Gyanodaya Prakashan, Nainital, India.

Singh JS, Tiwari AK, Saxena AK. 1985. Himalayan forest. A net source of carbon to the atmosphere. Environ Conserv 12: 67-69.

Singh SP, Rawat YS, Garkoti SC. 1997. Failure of broun oak (Quercus semicarpifolia) to regenerate in Central Himalaya: A case of environmental semi surprise. Curr Sci 73 (4): 371-374.

Sundriyal RC, Sharma E. 1996. Anthropogenic pressure on tree structure and biomass in the temperate forest of Mamlay Watershed in Sikkim. For Ecol Manag 81 (1-3): 113-134.

Ter Braak CJF, Prenrice IC. 1988. A theory of gradient analysis. Adv Ecol Res 18: 271-317.

Westman WE. 1975. Edaphic climax pattern of the pygmy forest region of California. Ecol Monogr 45: 109-135.

Wiart J. 1983. Ecosystem Villageois Traditionnel en Himalaya Nepalais: 1 Producton Forestiere Suffit-elle aux Besoins de la Population? [Dissertation]. Universite Scientifique et Medicale de Grenoble, France.

Woodwell GM. 1970. Effect of pollution on the structure and physiology of ecosystem. Science 168: 429-433. 